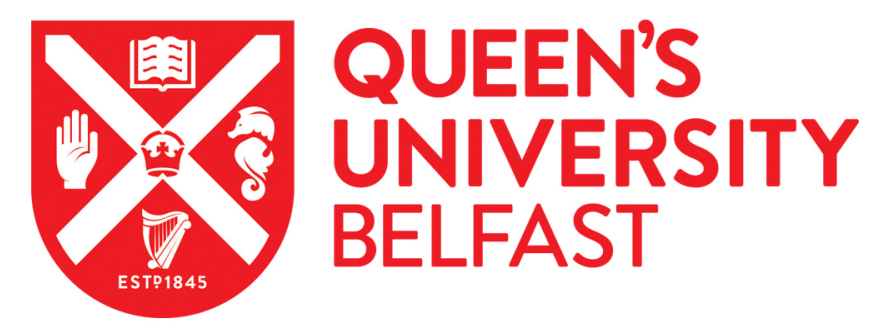

\title{
Association of C-Reactive Protein Genetic Polymorphisms With Late Age-Related Macular Degeneration.
}

European Eye (EUREYE) Study Group. (2017). Association of C-Reactive Protein Genetic Polymorphisms With Late Age-Related Macular Degeneration. JAMA Ophthalmology, 135(9), 909-916.

https://doi.org/10.1001/jamaophthalmol.2017.2191

Published in:

JAMA Ophthalmology

Document Version:

Peer reviewed version

Queen's University Belfast - Research Portal:

Link to publication record in Queen's University Belfast Research Portal

Publisher rights

Copyright 2017 American Medical Association. This work is made available online in accordance with the publisher's policies. Please refer to any applicable terms of use of the publisher.

\section{General rights}

Copyright for the publications made accessible via the Queen's University Belfast Research Portal is retained by the author(s) and / or other copyright owners and it is a condition of accessing these publications that users recognise and abide by the legal requirements associated with these rights.

Take down policy

The Research Portal is Queen's institutional repository that provides access to Queen's research output. Every effort has been made to ensure that content in the Research Portal does not infringe any person's rights, or applicable UK laws. If you discover content in the Research Portal that you believe breaches copyright or violates any law, please contact openaccess@qub.ac.uk. 
1 C-reactive protein $(C R P)$ genetic polymorphisms and late age-related macular

4 Valentina Cipriani, PhD, ${ }^{1,2,3,{ }^{*}}$ Ruth E. Hogg, $\mathrm{PhD}^{4,{ }^{*}}$ Reecha Sofat, $\mathrm{PhD}^{5}$ Anthony T. Moore, 5 FRCOphth ${ }^{1,2,6}$ Andrew R. Webster, FRCOphth ${ }^{1,2}$ John R.W. Yates, FRCP ${ }^{1,7}$ Astrid E. 6 Fletcher, $\mathrm{PhD}^{8}$ (on behalf of the EUREYE Study Group)

7

$8 \quad{ }^{1}$ UCL Institute of Ophthalmology, London, UK

$9 \quad{ }^{2}$ Moorfields Eye Hospital, London, UK

${ }^{3}$ UCL Genetics Institute, London, UK

${ }^{4}$ Centre for Public Health, Queen's University Belfast, UK

${ }^{5}$ Centre for Clinical Pharmacology, Division of Medicine, UCL, London, UK

${ }^{6}$ Ophthalmology Department, UCSF School of Medicine, San Francisco, CA, USA

${ }^{7}$ Department of Medical Genetics, University of Cambridge, UK

${ }^{8}$ Faculty of Epidemiology and Population Health, London School of Hygiene and Tropical Medicine, UK

*Valentina Cipriani and Ruth E. Hogg contributed equally to this work

Astrid E. Fletcher, the chair of the EUREYE Study Group confirms that members of the group are listed correctly.

Corresponding author and address for reprints: Valentina Cipriani, $\mathrm{PhD}$, UCL Institute of Ophthalmology, Department of Ocular Biology and Therapeutics, University College London, Wolfson Building ( $3^{\text {rd }}$ floor, Room 3/5), 11-43 Bath Street, London, EC1V 9EL, United Kingdom

\section{Manuscript text word count: 2985}


28 Key points

29 Question: Are variants in the C-reactive protein $(C R P)$ gene that are known to influence 30 circulating CRP concentration associated with late age-related macular degeneration (AMD)?

31 Findings: We selected four $C R P$ variants and conducted a pooled analysis of 1727 late AMD

32 cases and 1153 controls from two hospital-based case-control studies and one cross-sectional

33 population-based study. We found no statistical association between these $C R P$ variants and 34 any type of late AMD.

35 Meaning: Circulating CRP concentration is unlikely to be causally related to AMD. 
Abstract (Word count: 312)

37 Importance: C-reactive protein (CRP) is a circulating inflammatory marker associated with late age-related macular degeneration (AMD). It remains uncertain whether the association between CRP concentration and AMD is causal.

Objective: our primary objective was to assess whether $C R P$ single nucleotide polymorphisms (SNPs) that influence circulating CRP concentration are associated with late AMD.

Design, setting and participants: Participants in two UK hospital-based case-control studies (Cambridge and Moorfields) and one pan-European cross-sectional population-based study (EUREYE) were recruited between 2000 and 2006. Participants underwent dilated stereodigital fundus photography graded according to the International Classification of Agerelated Maculopathy and Macular Degeneration. There were 1727 cases of late AMD (1151 neovascular (nvAMD), 384 geographic atrophy (GA), 192 mixed (nvAMD and GA)), and 1153 controls. Early AMD cases $(n=574)$ were included only from EUREYE.

Exposure(s): Four common SNPs (rs1205, rs1130864, rs1800947, rs3093077) were selected based on demonstrated influence on circulating CRP circulation in the literature. In one study genotyping of rs3093077 failed and rs1800947 was only typed in one study.

Main Outcome and Measure(s): Genetic multiplicative model for the association of SNPs with late AMD adjusted for age and sex and expressed as Odds Ratios (OR) and 95\% Confidence Intervals (95\% CI).

Results: The mean age (SD) was 78.7 (7.4) for late AMD cases (of which 668 male, 38.7\%) and 74.9 (7.0) for controls (of which 510 male, 44.2\%). In the pooled results of all three studies, neither rs1205 nor rs1130864 were associated with late AMD, per allele OR=0.99 
60 phenotype were: $\mathrm{GA}, \mathrm{OR}=0.91(95 \% \mathrm{CI}=0.74-1.13)$ and $\mathrm{OR}=0.94 \quad(95 \% \mathrm{CI}=0.76-1.16)$;

61 nvAMD, $\mathrm{OR}=1.01(95 \% \mathrm{CI}=0.87-1.19)$ or $\mathrm{OR}=0.99(95 \% \mathrm{CI}=0.84-1.16)$. There was no

62 association of rs3093077 and rs1800947 with late AMD or any late AMD phenotype. There

63 were no significant findings for early AMD.

64 Conclusions and relevance: Our results do not support a causal association between CRP 65 concentration and AMD. 
Introduction

67

68

69

Inflammation has been recognised to play a key role in age-related macular degeneration $(A M D)^{1,2}$ strengthened by the discovery of multiple genetic risk loci involved in the complement pathway. ${ }^{3-7}$ The early and intermediate stages of AMD are characterised by drusen which are accumulations of lipids and proteins that form discrete deposits under the retinal pigment epithelium, and these can both initiate and reflect inflammatory reactions within the complement pathway. ${ }^{1}$ Several studies have investigated the relationship between circulating inflammatory markers and the onset and progression of $\mathrm{AMD}^{8-12}$ both to understand the pathogenesis of AMD and also to assess their potential as prognostic biomarkers. One such inflammatory marker is C-reactive protein (CRP), an acute phase reactant ${ }^{13}$ which has been shown in two meta-analyses to be associated with late AMD. ${ }^{14,15}$ CRP is involved in the initiation and modulation of the classical complement pathway as well as the alternative complement pathway through binding with complement factor $\mathrm{H}(\mathrm{CFH})$. Therefore it could be postulated that high concentrations of CRP have a causal role in the development of AMD by altering complement-related inflammatory responses to drusen.

Demonstration of a causal role of CRP is problematic as is illustrated by the abundant work carried out on CRP and coronary heart disease (CHD). Despite the numerous studies (including many prospective studies) consistently showing an increased risk of CHD with increasing CRP, ${ }^{16}$ no studies have demonstrated an association between single nucleotide polymorphisms (SNPs) in the CRP gene and risk of CHD. ${ }^{17,18}$ This approach, described as Mendelian randomisation (MR), ${ }^{19}$ showed that, although certain $C R P$ SNPs have been shown to influence the concentrations of circulating CRP, ${ }^{20}$ the lack of a relationship between those CRP SNPs and CHD suggests that CRP is unlikely to have a causal influence on CHD. ${ }^{17} \mathrm{~A}$ key point in the MR approach is that the causal role of a risk exposure for a specific disease is 
assessed testing the relationship between genetic variants (that have been reliably shown to influence the exposure) and the disease of interest. The MR approach avoids bias due to reverse causation because genotypes are fixed at conception and therefore are not subject to confounding nor modification by the onset of disease. ${ }^{21}$

Three previous studies have investigated the relationship between $C R P$ genetic variants and AMD but none have found an association. ${ }^{22-24}$ The total number of late AMD cases in the three studies was 394 and each study may have lacked power. We investigated the relationship between AMD and $C R P$ polymorphisms in three studies: the European Eye study (EUREYE), a cross-sectional population-based epidemiological study of AMD prevalence and risk factors, ${ }^{25}$ and two hospital-based AMD case-control studies, the Cambridge ${ }^{7}$ and Moorfields Eye Hospital (MEH) ${ }^{26}$ AMD studies.

\section{Methods}

For all three studies ethical approval was obtained from either national or local ethics committees and adhered to the tenets of the Declaration of Helsinki. All participants provided written informed consent to undergo the clinical examination and epidemiological data collection, and to provide a blood sample for biochemical and genetic analyses.

\section{EUREYE Study}

Participants were recruited between 2001 and 2002 by random sampling of the population aged 65 years and older in seven centers: Bergen (Norway), Tallinn (Estonia), Belfast (United Kingdom), Paris-Creteil (France), Verona (Italy), Thessaloniki (Greece) and Alicante (Spain). Methods have been published in full elsewhere. ${ }^{25}$ Trained field workers administered structured questionnaires including smoking, diet and sunlight exposure and self-reported 
medical history. The ophthalmic examination included dilated stereo digital fundus photography. Stereo fundus images were graded at a single reading centre (Erasmus University Rotterdam) according to the International Classification of Age-related Maculopathy and Macular Degeneration ${ }^{27}$ and then categorized into five mutually exclusive grades. Grade 0 was defined as a macula free of drusen or pigmentary irregularities or with hard drusen $(<63 \mu \mathrm{m})$ only. Early AMD was subdivided in grade 1, defined as soft distinct drusen $(\geq 63 \mu \mathrm{m})$ or pigmentary abnormalities, grade 2 as soft indistinct drusen $(\geq 125 \mu \mathrm{m})$ or reticular drusen only or soft distinct drusen $(\geq 63 \mu \mathrm{m})$ with pigmentary abnormalities, and grade 3 as soft indistinct drusen $(\geq 125 \mu \mathrm{m})$ or reticular drusen with pigmentary abnormalities. Grade 4 was defined as presence of either neovascular AMD (nvAMD) (presence of any of the following: serous or hemorrhagic retinal or retinal pigment epithelial detachment, subretinal neovascular membrane, periretinal fibrous scar) or geographic atrophy (GA) (well-demarcated area of retinal pigment atrophy with visible choroidal vessels). In the present analysis, for reason of costs, a random sample of controls (AMD stage 0) was frequency matched on age (within a year), sex and centre with cases with (i) late AMD (nvAMD or GA) and (ii) cases with early AMD (AMD stages 2 and 3). Non-fasting venous blood samples were collected at the ophthalmological examination, separated within 4 hours of collection and serum was stored at $-20^{\circ} \mathrm{C}$ for up to 4 weeks before frozen samples were transferred to a single laboratory (Queens University Belfast) for storage at $-80^{\circ} \mathrm{C}$.

Cambridge AMD study

The Cambridge AMD study is a case-control study of AMD with participants recruited from ophthalmic clinics in London, the South East of England and the North West of England between 2002 and $2006 .^{7}$ All cases had at least one eye affected by nvAMD and/or GA. 
evidence of other inflammatory or retinovascular disease such as retinal vessel occlusion, diabetic retinopathy or chorioretinitis that could contribute to the development or confound the diagnosis of maculopathy. Almost all the controls were spouses or partners of index cases and the remainder were friends of cases. All participants described themselves as "white" rather than "other" on a recruitment questionnaire. Participants were examined by an ophthalmologist and health, lifestyle and smoking data were collected, and underwent colour stereoscopic fundus photography of the macular region. Images were graded at the Reading related Maculopathy and Macular Degeneration. ${ }^{27}$ Blood was drawn at the time of interview; EDTA samples were obtained for DNA extraction, and lithium-heparin plasma samples stored at $-80^{\circ} \mathrm{C}$ were later used for CRP measurements.

MEH AMD study

The MEH AMD study is a case-control study of AMD undertaken at Moorfields Eye Hospital, London between 2001 and 2004. ${ }^{26}$ Only cases and controls of European origin were included in the study. Cases consisted of patients attending routine follow up appointments and all new referrals from primary care clinics, casualty, and other centres with any type of AMD to try to limit any selection bias. Cases were excluded if they had retino-choroidal inflammatory disease, diabetic retinopathy, branch retinal vein or artery occlusion or any other cause of visual loss other than amblyopia. Controls were recruited from spouses or friends of cases, or were from local residential homes for the elderly within 5 miles of the hospital. Each participant was interviewed specifically for the study, and a family history, smoking history and other medical history was taken. The ophthalmic examination included Snellen acuity, slit-lamp examination and bio-microscopic funduscopy. All participants had colour stereoscopic fundus photography of the macular region and the images were graded at 
the Reading Centre, Moorfields Eye Hospital, London using the International Classification of Age-related Maculopathy and Macular Degeneration. ${ }^{27}$ Controls were excluded if drusen >63um were evident. A sample of peripheral blood was obtained from each participant and stored at $-20^{\circ} \mathrm{C}$ for DNA extraction and $-80^{\circ} \mathrm{C}$ for analyte measurement.

\section{Circulating $C$-reactive protein concentration}

Circulating CRP concentration was measured in the EUREYE and Cambridge AMD studies.

In the EUREYE study, the concentration of serum CRP was assessed by a high-sensitivity latex-enhanced turbidometric immunoassay (Wako Chemicals $\mathrm{GmbH}$, Neuss, Germany) using a Cobas Fara analyzer (Roche Diagnostics, Burgess Hill, United Kingdom). In the Cambridge AMD study, the concentration of plasma CRP was measured by high sensitivity particle enhanced immunonephelometric assay (Siemens CardioPhase hsCRP) using a Siemens Dade Behring BNII Nephelometer. Participants with CRP values >10mg/L indicative of acute infection ${ }^{28}$ were not included in the analysis ( 90 from EUREYE and 51 from Cambridge). CRP concentration was natural logarithmically transformed to ensure normality of the distribution, and further $\mathrm{Z}$ transformed for investigation of the association of a one standard deviation (SD) increase in log CRP with AMD.

Genotyping

We chose four common SNPs (rs1205, rs1130864 and rs1800947 in the 3' UTR, and synonymous rs3093077 in exon 2) in the $C R P$ gene previously reported in a meta-analysis on CRP and coronary heart disease using a MR approach. ${ }^{17}$ These variants were selected as the most parsimonious set of tagging SNPs capturing maximum haplotype diversity and of demonstrated association with CRP concentrations among subjects of European descent. ${ }^{21,29}$ 
using KASPar chemistry, a competitive allele specific polymerase chain reaction (PCR) SNP genotyping system using FRET quencher cassette oligos. Genotyping in the Cambridge AMD study was carried out using the ABI PRISM SNaPshot ddNTP Primer Extension Kit and a 3100 Genetic Analyser (Applied Biosystems, Foster City, CA). Genotyping in the MEH AMD study was carried out using a Taqman (ABI) assay (Applied Biosystems, Foster City, CA). Departure from Hardy-Weinberg equilibrium was assessed for all SNPs in control participants using a $\chi^{2}$ goodness-of-fit test.

\section{Statistical association analysis}

We investigated the association of $C R P$ genotype with late AMD and by late AMD phenotype (GA, nvAMD). We also report results for early AMD (available in the EUREYE study only). All association analyses were conducted as two-stage, fixed-effects metaanalysis of the available individual participant data from the three studies. Heterogeneity across study centres was assessed using the $\mathrm{I}^{2}$ statistic. We calculated odds ratios (OR) and their corresponding 95\% confidence intervals (CI) using logistic regression models adjusted for sex and age. ORs were expressed as per one minor allele (multiplicative genetic model).

In order to confirm that, in our study population, the selected SNP genotypes influence circulating CRP concentrations, we examined the association of $C R P$ genotype with CRP concentration in controls via a Wald test on the genotype coded as $0,1,2$ according to the number of minor alleles using linear regression models adjusted for age and sex. We report geometric means with standard deviations (SD) for concentrations by genotype.

For comparison with previous studies, we analysed the association of CRP concentration with late AMD and late AMD phenotype using logistic regression models. These analyses were adjusted for age and sex and additionally for a priori confounders including history of 
myocardial infection or stroke (CVD), diabetes and smoking. ORs were expressed as per one

215

216

217 SD change of log CRP concentration.

All statistical analyses were conducted using Stata software (Version13.1, Stata Corp LP College Station, Texas, USA) and ipdmetan and mvmeta commands were used for conducting meta-analyses of individual participant data.

\section{Results}

Characteristics of participants and genotype frequencies by study are shown in Table1. Genotyping of rs3093077 failed in the EUREYE study and rs1800947 was not typed in the MEH and Cambridge AMD studies. In each study, no deviation from Hardy-Weinberg equilibrium was observed for any SNP and control minimum allele frequencies (MAF) were similar both between studies and to those reported for European ancestry in the HapMap CEU samples: rs1205 MAF (T) (0.34), rs1130864 MAF (T) (0.31), rs3093077 MAF (G) (0.05), rs1800947 MAF (C) (0.07). There were significant associations in the pooled analysis for rs $1205 \mathrm{~T}$ allele with lower CRP concentration $(\mathrm{p}=0.001)$ and for rs1130864 $\mathrm{T}$ allele with higher CRP ( $\mathrm{p}=0.014)$ in controls (Table 2). For the two rare SNPs rs3093077 and rs1800947 there was no association with CRP concentrations in controls.

There was no evidence of any association of CRP SNPs rs1205, rs1130864 or rs3093077 with late AMD, or with type of late AMD, either from the individual studies or in the metaanalysis, or for SNP rs1800947 in the EUREYE study (Table 3).

A significant association was observed in the pooled age and sex adjusted analysis of CRP concentration with late AMD (Table 4). Additional adjustment for smoking, CVD and diabetes slightly reduced the ORs (from 1.19 to 1.16 ) and the $\mathrm{p}$ value went from 0.03 to 0.07 . 
257 None of the three previous studies that investigated $C R P$ polymorphisms and AMD have 258

Analyses of CRP concentration by type of late AMD were OR=1.35, 95\% CI (1.03-1.76), $\mathrm{p}=0.03$ for GA and $1.09(0.91-1.29), \mathrm{p}=0.36$ for nvAMD.

Only the EUREYE study had population based data available on early AMD. There was no association of CRP concentration with early AMD, in age and sex adjusted or fully adjusted analyses, $\mathrm{OR}=1.01,95 \% \mathrm{CI}(0.82-1.25), \mathrm{p}=0.90$ or of any $C R P$ SNPs with early AMD, OR $(95 \% \mathrm{CI}) \mathrm{p}$ values for a multiplicative genetic model were: $\mathrm{rs} 1205, \mathrm{OR}=1.08(0.98-1.20)$ $\mathrm{p}=0.13 ; \mathrm{rs} 1130864, \mathrm{OR}=0.89(0.75-1.06), \mathrm{p}=0.20 ; \mathrm{rs} 1800947, \mathrm{OR}=1.20(0.89-1.63), \mathrm{p}=0.23$.

\section{Discussion}

In this meta-analysis of three studies of AMD including 1727 cases with late AMD and 1153 controls, we found no evidence from analysis of $C R P$ variants to support a causal association of CRP concentration with late AMD, either nvAMD or GA.

In agreement with many other studies, ${ }^{17,18,30}$ we found that the $\mathrm{T}$ allele of rs1205 was associated with lower CRP concentrations in controls. However there was no protective association between the $\mathrm{T}$ allele and either nvAMD or GA in any of the three studies. Similarly the $\mathrm{T}$ allele of rs1130864 was associated with higher $\mathrm{CRP}^{17}$ concentrations in controls but the T allele was not associated with higher odds of either late AMD phenotype. found an association. ${ }^{22-24}$ These studies had smaller sample size than our investigation and included two ${ }^{23}$ or three ${ }^{22,24}$ of the SNPs we investigated (rs1205, rs1130864, rs 1800947). In a case-control study (111 mixed early and late cases and 401 controls) nested within the 
prospective follow-up of the Physicians Health Study, AMD risk was not influenced by any

262

263

264

265

266

267

268

269

270

271

272

273

274

275

276

277

278

279

280

281

282

283 of seven CRP SNPs ${ }^{24}$ nor for any of nine CRP SNPs in a sib-pair study of 112 cases of nvAMD. ${ }^{22}$ The Rotterdam study (5,861 subjects, of whom 171 late cases) suggested that $C R P$ haplotypes may modify the association of $C F H Y 402 H$ genotype with late AMD but the $C R P$ haplotypes did not influence AMD risk directly; results were not presented for the three $C R P$ SNPs individually. ${ }^{23}$ Additionally, a meta-analysis of 15 genome-wide association studies $(\text { GWAS })^{31}$ and a recent large GWAS (16,144 cases and 17,832 controls) of AMD did not show any genome-wide significant association signals at the $C R P$ locus. ${ }^{32}$

In our fully adjusted association analyses between CRP concentration and AMD, we did not find a significant association with all late AMD ( $\mathrm{p}=0.07 ; 1,324$ subjects, of whom 489 cases) and nvAMD ( $\mathrm{p}=0.36 ; 318$ cases) or with early AMD ( $\mathrm{p}=0.90 ; 574$ cases) which has been reported in other studies. ${ }^{9,15,33}$ The association with AMD is not consistent, particularly in studies investigating early AMD. ${ }^{11,34-37}$ In a large meta-analysis comparing low $(<1 \mathrm{mg} / \mathrm{L})$ versus high $(>3 \mathrm{mg} / \mathrm{L}) \mathrm{CRP}$ concentrations $($ SI units $(<9.5 \mathrm{nmol} / \mathrm{L})(>28.6 \mathrm{nmol} / \mathrm{L})$, Hong et al. ${ }^{14}$ reported an OR of 1.30 (95\% CI 1.03-1.65) in three studies that combined early and late AMD (including 4,522 subjects, of whom 1,629 cases), and an OR of 2.19 (95\% CI 1.383.47) in eight studies of late AMD (including 35,168 subjects, of whom 1,603 cases). Estimates for late AMD varied considerably according to method of AMD ascertainment with much higher ORs reported for clinical diagnosis $(\mathrm{OR}=3.8)$ compared to fundus photography $(\mathrm{OR}=1.4)$, adjustment for diabetes $(\mathrm{OR}=1.6)$ compared to no adjustment $(\mathrm{OR}=2.5)$ and study design. Population-based cross-sectional or case-control studies had lower ORs than clinic case-control studies or longitudinal studies. In a pooled analysis of prospective nested case-control studies from five population based US cohorts (647 cases of 
whom 190 were nvAMD), concentrations of CRP >3mg/L compared to $<1 \mathrm{mg} / \mathrm{L}$ were associated with risk of all late AMD and nvAMD. ${ }^{15}$

287

While our study was larger than the three previous studies that investigated the relationship between $C R P$ variants and AMD, it does have a number of limitations, especially with respect to the relationship between CRP concentration and AMD. In the EUREYE and Cambridge studies, blood used for measuring CRP concentration was collected at the same time as ascertainment of AMD and therefore we cannot exclude reverse causation, i.e., an inflammatory response, for example due to risk factors for AMD such as atherosclerosis and cardiovascular disease. We only had one measure of CRP and this may not represent longerterm usual concentrations. Additionally, circulating CRP may not adequately reflect the CRP concentration in the relevant tissues. It is notable that the monomeric form of CRP (mCRP) has been shown to be responsible for upregulation of proinflammatory cytokines, IL- 8 and CCL2 in retinal pigment epithelial cells and that patients with AMD carrying the risk variants of $C F H$ Y204H showed impaired binding of mCRP. ${ }^{38}$

Genetic variants in $C R P$ are not subject to reverse causation ${ }^{19,21}$ and therefore provide stronger evidence on the possible association of CRP concentration with AMD. We investigated only a small number of $C R P$ SNPS. Nevertheless, we selected SNPs expected to show a causal relationship with AMD, since these SNPs influence CRP concentrations. ${ }^{17,20,21,29}$ Variants associated with CRP concentrations in other loci have been identified in large GWAS and replication studies. ${ }^{18,39}$ These variants are in genes primarily involved in lipid, metabolic or immune pathways and include IL6R, HNF1A, APOCI. In a GWAS of coronary heart disease (CHD) the APOC1 variant (rs4420638) was associated with increased risk of CHD but in an opposite direction to that predicted from its relationship with 
310 CRP and consistent with its effects on LDL and HDL cholesterol and triglycerides. ${ }^{18}$ This

311 variant has also been identified at genome-wide significance to increase AMD risk. ${ }^{31}$ APOC1

312 is part of the apolipoprotein gene cluster which plays a major role in lipid transport and

313 metabolism and includes the well established APOE AMD risk variant (rs429358). ${ }^{40}$ Linkage

314 Disequilibrium $\left(\mathrm{r}^{2}=0.7\right)$ is observed between $\mathrm{rs} 4420638$ and $\mathrm{rs} 429358$ in the EUR

315 populations from the Phase 3 of the 1000 Genomes Project

316 (http://analysistools.nci.nih.gov/LDlink accessed Oct $3^{\text {rd }}$ 2016).

317

318 In summary, from the analogy with $\mathrm{CHD}^{17}$ and the identification of alternate genetic loci for

319 CRP concentrations discussed above, along with the lack of association of variants in the

320 CRP locus with AMD shown in our meta-analysis corroborating results from previous

321 studies, we conclude that CRP concentration is unlikely to be causally related to AMD. 
Table 1. Demographic, C-reactive protein (CRP) concentration and CRP SNP genotype frequencies by study.

\begin{tabular}{|c|c|c|c|c|c|c|c|}
\hline & \multicolumn{3}{|c|}{ EUREYE } & \multicolumn{2}{|c|}{ Cambridge } & \multicolumn{2}{|c|}{$\mathrm{MEH}$} \\
\hline & Controls & Early AMD & Late AMD & Controls & Late AMD & Controls & Late AMD \\
\hline $\mathrm{N}$ & 643 & 574 & 152 & 262 & 427 & 245 & 1145 \\
\hline $\mathrm{Age}^{1}$ & $74.7(6.1)$ & $74.6(5.9)$ & $79.5(7.2)$ & $75.7(7.8)$ & $80.4(6.9)$ & $74.8(8.0)$ & $77.9(7.4)$ \\
\hline Male (\%) & 46.8 & 48.6 & 34.2 & 40.1 & 45.9 & 41.9 & 36.6 \\
\hline $\mathrm{CRP}^{2}$ & $1.88(1.21)$ & $1.99(1.35)$ & $1.90(1.28)$ & $1.84(1.72)$ & $2.15(1.92)$ & \multicolumn{2}{|c|}{ Not available } \\
\hline \multicolumn{8}{|l|}{ rs1205 } \\
\hline $\mathrm{CC}$ & $273(44.6)$ & $246(43.9)$ & $58(39.7)$ & $119(46.5)$ & $200(47.7)$ & $101(43.7)$ & $478(44.1)$ \\
\hline $\mathrm{CT}$ & 267 (43.6) & $240(42.9)$ & $75(51.4)$ & $113(44.1)$ & $183(43.7)$ & $107(46.3)$ & $507(46.8)$ \\
\hline TT & $72(11.8)$ & $74(13.2)$ & $13(8.9)$ & $24(9.4)$ & $36(8.6)$ & $23(10.0)$ & $99(9.1)$ \\
\hline Total & 612 & 560 & 146 & 256 & 419 & 231 & 1084 \\
\hline $\mathrm{HWE}^{3}$ & 0.36 & & & 0.70 & & 0.49 & \\
\hline MAF $^{4}$ & 0.35 & & & 0.31 & & 0.33 & \\
\hline \multicolumn{8}{|l|}{ rs1130864 } \\
\hline $\mathrm{CC}$ & $280(45.9)$ & 265 (47.6) & $68(46.3)$ & $126(48.1)$ & $188(44.4)$ & $104(44.6)$ & $528(48.7)$ \\
\hline $\mathrm{CT}$ & $266(43.6)$ & $245(44.0)$ & $68(46.3)$ & $113(43.1)$ & $204(48.2)$ & 108 (46.4) & $451(41.6)$ \\
\hline TT & $64(10.5)$ & $47(8.4)$ & $11(7.4)$ & $23(8.8)$ & $31(7.3)$ & $21(9.0)$ & $105(9.7)$ \\
\hline Total & 610 & 557 & 147 & 262 & 423 & 233 & 1084 \\
\hline $\mathrm{HWE}^{3}$ & 0.73 & & & 0.74 & & 0.35 & \\
\hline MAF $^{4}$ & 0.35 & & & 0.30 & & 0.32 & \\
\hline rs3093077 & \multicolumn{3}{|c|}{ Failed } & & & & \\
\hline TT & & & & $232(88.5)$ & $380(89.4)$ & $211(90.6)$ & $960(88.5)$ \\
\hline GT & & & & $30(11.5)$ & 45 (10.6) & $21(9.0)$ & $119(11.0)$ \\
\hline GG & & & & 0 & 0 & $1(0.4)$ & $6(0.5)$ \\
\hline Total & & & & 262 & 425 & 233 & 1085 \\
\hline HWE $^{3}$ & & & & 0.33 & 16 & 0.55 & \\
\hline MAF $^{4}$ & & & & 0.06 & & 0.05 & \\
\hline
\end{tabular}




\begin{tabular}{|r|c|c|c|c|c|c|c|}
\hline rs1800947 & & & & \multicolumn{2}{|c|}{ Not available } & \multicolumn{2}{|c|}{ Not available 323} \\
\hline GG & $519(88.7)$ & $471(87.4)$ & $113(83.1)$ & & & 324 \\
\hline CG & $63(10.8)$ & $65(12.1)$ & $21(15.4)$ & & & & 325 \\
\hline CC & $3(0.5)$ & $3(0.6)$ & $2(1.5)$ & & & & 326 \\
\hline Total & 585 & 539 & 136 & & & & 327 \\
\hline HWE $^{3}$ & 0.50 & & & & & & \\
\hline MAF $^{4}$ & 0.07 & & & & & & 328 \\
\hline
\end{tabular}

$329{ }^{1}$ Mean (Standard Deviation)

$330{ }^{2}$ Geometric mean (Standard Deviation) (mg/dL)

$331{ }^{3}$ Hardy Weinberg Equilibrium p value

$332 \quad{ }^{4}$ Minimum Allele Frequency

333 
334 Table 2. C-reactive protein (CRP) concentration by genotypes of CRP SNPs in controls.

335

\begin{tabular}{|c|c|c|c|c|}
\hline & \multicolumn{2}{|c|}{ EUREYE } & \multicolumn{2}{|c|}{ Cambridge } \\
\hline$C R P$ SNPs & $\mathrm{N}$ & $\mathrm{CRP}^{1}$ & $\mathrm{~N}$ & $\begin{array}{ll}\text { CRP }^{1} & \\
& 337\end{array}$ \\
\hline $\mathrm{rs} 1205$ & & & & \\
\hline $\mathrm{CC}$ & 255 & $2.05(1.36)$ & 105 & $1.97(1.83)^{38}$ \\
\hline TC & 249 & $1.79(1.10)$ & 96 & $1.82(1.7339$ \\
\hline TT & 67 & $1.65(1.01)$ & 21 & $1.28(1.25)$ \\
\hline $\mathrm{p}^{2}$ & & 0.003 & & $0.128 \quad 340$ \\
\hline p pooled ${ }^{3}$ & \multicolumn{4}{|c|}{0.001} \\
\hline rs1130864 & & & & \\
\hline $\mathrm{CC}$ & 264 & $1.87(1.24)$ & 110 & $1.43(1.42)$ \\
\hline TC & 248 & $1.84(1.09)$ & 94 & $2.32(2.1343$ \\
\hline TT & 56 & $2.26(1.65)$ & 21 & $2.19(1.29)_{44}$ \\
\hline $\mathrm{p}^{2}$ & & 0.185 & & 0.002 \\
\hline p pooled $^{3}$ & \multicolumn{4}{|c|}{0.014} \\
\hline rs3093077 & & & & 346 \\
\hline TT & & & 200 & $1.82(1.72)$ \\
\hline GT & & & 25 & $1.83(1.99)^{4 /}$ \\
\hline GG & & & 0 & \\
\hline $\mathrm{p}^{2}$ & & & & 0.829 \\
\hline rs1800947 & & & & 345 \\
\hline GG & 486 & $1.87(1.20)$ & & 350 \\
\hline GC & 56 & $2.15(1.30)$ & & \\
\hline $\mathrm{CC}$ & 3 & $1.11(0.74)$ & & \\
\hline $\mathrm{p}^{2}$ & & 0.455 & & 352 \\
\hline
\end{tabular}

353

$354 \quad{ }^{1}$ Geometric mean (Standard Deviation) $\mathrm{mg} / \mathrm{dL}$

$355{ }^{2} \mathrm{p}$ value from per allele regression model of age and sex adjusted log serum CRP in each study

$356{ }^{3} \mathrm{p}$ value from per allele regression model of age and sex adjusted log serum CRP in meta-analysis 
Table 3. Association of CRP SNPS with late AMD in the EUREYE, Cambridge and MEH AMD studies.

\begin{tabular}{|c|c|c|c|c|}
\hline & EUREYE & Cambridge & MEH & Pooled \\
\hline & OR $(95 \% \mathrm{CI}) \mathrm{p}$ value $^{1}$ & OR $(95 \% \mathrm{CI}) \mathrm{p}$ value $^{1}$ & OR $(95 \% \mathrm{CI}) \mathrm{p}$ value $^{1}$ & OR $(95 \% \mathrm{CI}) \mathrm{p}$ value $\mathrm{e}^{1}$ \\
\hline Late AMD & & & & \\
\hline rs1205 & $1.06(0.80-1.40) \mathrm{p}=0.69$ & $0.95(0.73-1.22) \mathrm{p}=0.66$ & $0.98(0.78-1.23) \mathrm{p}=0.87$ & $0.99(0.86-1.14) \mathrm{p}=0.88$ \\
\hline rs1130864 & $0.86(0.64-1.16) \mathrm{p}=0.33$ & $1.17(0.90-1.52) \mathrm{p}=0.24$ & $0.89(0.71-1.11) \mathrm{p}=0.29$ & $0.96(0.83-1.11) \mathrm{p}=0.60$ \\
\hline rs3093077 & Not available & $0.92(0.55-1.54) \mathrm{p}=0.76$ & $1.26(0.80-1.98) \mathrm{p}=0.32$ & $1.10(0.78-1.55) \mathrm{p}=3 \odot 1$ \\
\hline \multirow[t]{2}{*}{ rs1800947 } & $1.54(0.95-2.50) \mathrm{p}=0.08$ & Not available & Not available & \\
\hline & & & & 362 \\
\hline \multicolumn{5}{|l|}{ Neovascular AMD } \\
\hline rs1205 & $1.04(0.75-1.44) \mathrm{p}=0.81$ & $0.99(0.76-1.30) \mathrm{p}=0.96$ & $1.02(0.80-1.29) \mathrm{p}=0.88$ & $1.01(0.87-1.19) \mathrm{p}=3.85$ \\
\hline rs1130864 & $0.83(0.58-1.17) \mathrm{p}=0.28$ & $1.29(0.97-1.71) \mathrm{p}=0.08$ & $0.90(0.71-1.13) \mathrm{p}=0.35$ & $0.99(0.84-1.16) \mathrm{p}=0.86$ \\
\hline rs3093077 & Not available & $1.02(0.59-1.77) \mathrm{p}=0.95$ & $1.25(0.78-2.01) \mathrm{p}=0.35$ & $1.15(0.80-1.64) p=0.43$ \\
\hline rs1800947 & $1.68(0.97-2.93) \mathrm{p}=0.06$ & Not available & Not available & 365 \\
\hline Geographic Atrophy & & & & 366 \\
\hline rs1205 & $1.11(0.71-1.74) \mathrm{p}=0.65$ & $0.91(0.60-1.38) \mathrm{p}=0.65$ & $0.85(0.64-1.13) \mathrm{p}=0.26$ & $0.91(0.74-1.13) \mathrm{p}=0.40$ \\
\hline rs1130864 & $0.98(0.61-1.60) \mathrm{p}=0.95$ & $1.18(0.79-1.76) \mathrm{p}=0.42$ & $0.83(0.62-1.10) \mathrm{p}=0.20$ & $0.94(0.76-1.16) \mathrm{p}=8.68$ \\
\hline rs3093077 & Not available & $0.73(0.29-1.84) \mathrm{p}=0.51$ & $1.62(0.94-2.77) \mathrm{p}=0.08$ & $1.31(0.83-2.10) \mathrm{p}=0.25$ \\
\hline rs1800947 & $1.37(0.62-3.04) \mathrm{p}=0.43$ & Not available & Not available & 368 \\
\hline
\end{tabular}

369

$370{ }^{1}$ Sex- and age-adjusted Odds Ratio (OR), 95\% Confidence Interval (CI) and p value for a genetic multiplicative model. 
Table 4. Association of C-Reactive protein (CRP) concentration with late age-related macular degeneration (AMD).

\begin{tabular}{|c|c|c|c|c|c|c|c|c|c|}
\hline & \multicolumn{3}{|c|}{ Late AMD } & \multicolumn{3}{|c|}{ Neovascular AMD } & \multicolumn{3}{|c|}{ Geographic Atrophy } \\
\hline & EUREYE & Cambridge & $\begin{array}{l}\text { Pooled } \\
\mathrm{N}=1324\end{array}$ & EUREYE & Cambridge & $\begin{array}{l}\text { Pooled } \\
\mathrm{N}=1153\end{array}$ & EUREYE & Cambridge & $\begin{array}{l}\text { Pooled } \\
\mathrm{N}=946\end{array}$ \\
\hline $\mathrm{OR}^{1,2}$ & 1.17 & 1.20 & 1.19 & 1.09 & 1.13 & 1.12 & 1.33 & 1.48 & 1.43 \\
\hline $95 \% \mathrm{CI}$ & $0.89-1.56$ & $1.00-1.45$ & $1.02-1.39$ & $0.79-1.51$ & $0.93-1.38$ & $0.95-1.33$ & $0.84-2.09$ & $1.09-2.02$ & $1.11-1.84$ \\
\hline $\mathrm{p}^{3}$ & & & 0.026 & & & 0.189 & & & 0.006 \\
\hline $\mathrm{OR}^{4}$ & 1.10 & 1.18 & 1.16 & 1.00 & 1.12 & 1.09 & 1.26 & 1.39 & 1.35 \\
\hline $95 \% \mathrm{CI}$ & $0.82-1.47$ & $0.98-1.43$ & $0.99-1.36$ & $0.71-1.41$ & $0.91-1.37$ & $0.91-1.29$ & $0.79-2.03$ & $1.01-1.92$ & $1.03-1.76$ \\
\hline $\mathrm{p}$ & & & 0.068 & & & 0.361 & & & 0.029 \\
\hline
\end{tabular}

372

$373{ }^{1}$ Odds Ratio per Standard Deviation change in log CRP concentration

$374 \quad 2$ adjusted for age and sex

$375{ }^{3} \mathrm{p}$ value from meta-analysis of EUREYE and Cambridge

$376{ }^{4}$ adjusted for age, sex, diabetes, cardiovascular disease, smoking 
378 For the Cambridge AMD study we gratefully acknowledge help with patient recruitment 379 from members of the Genetic Factors in AMD Study Group (Yates JRW et al., N Engl J Med 2007; 357:553-561). We thank Jane Khan and Humma Shahid for expert clinical evaluation and sampling of the study participants. For the Moorfields Eye Hospital AMD study we thank Sharon A. Jenkins for patient recruitment. EUREYE was supported by the European Commission Vth Framework (QLK6-CT-1999-02094) with additional funding for cameras provided by the Macular Disease Society UK, for DNA extraction and genotyping (MRC Biomarkers Award G0601354). The Cambridge AMD study was funded by the Medical Research Council (grant G0000067). The Moorfields Eye Hospital AMD study was partly supported by Fight for Sight including the Mercer Fund. VC is funded by National Institute for Health Research Biomedical Research Centre at Moorfields Eye Hospital National Health Service Foundation Trust and UCL Institute of Ophthalmology. RS was funded by a British Heart Foundation (Schillingford) Clinical Research Training Fellowship (FS/07/011). The funding sources had no role in the design and conduct of the study; collection, management, analysis, and interpretation of the data; preparation, review, or approval of the manuscript; and decision to submit the manuscript for publication. The views expressed in this publication are those of the authors and not necessarily those of the funding agencies. VC and AEF had full access to all the data in the study and takes responsibility for the integrity of the data and the accuracy of the data analysis.

The EUREYE Study Group (Augood CA et al., Arch Ophthalmol 2006; 124:529-535)

Astrid Fletcher, London School of Hygiene \& Tropical Medicine, UK

401

Graham Bentham, University of East Anglia, Norwich, UK

402 Usha Chakravarthy, Queen's University Belfast, UK

403

Paulus TVM de Jong, Netherlands Institute for Ophthalmic Research, Amsterdam,

404 Netherlands

405 Mati Rahu, National Institute for Health Development, Tallinn, Estonia

407 Gisele Soubrane, Universitaire de Créteil, Paris, France 
408 Laura Tomazzoli, Università degli studi di Verona, Italy

409 Fotis Topouzis, Aristotle University of Thessaloniki, Greece

410 Jesus Vioque, Universidad Miguel Hernandez, Alicante, Spain

411 Ian Young, Queen's University Belfast, UK

412

413 Conflict of interest

414 No conflicting relationship exists for any author. 
1. Hageman GS, Luthert PJ, Victor Chong NH, Johnson LV, Anderson DH, Mullins RF. An integrated hypothesis that considers drusen as biomarkers of immune-mediated processes at the RPE-Bruch's membrane interface in aging and age-related macular degeneration. Prog Retin Eye Res. 2001;20(6):705-732.

2. Anderson DH, Mullins RF, Hageman GS, Johnson LV. A role for local inflammation in the formation of drusen in the aging eye. Am J Ophthalmol. 2002;134(3):411-431.

3. Edwards AO, Ritter R, 3rd, Abel KJ, Manning A, Panhuysen C, Farrer LA. Complement factor H polymorphism and age-related macular degeneration. Science. 2005;308(5720):421-424.

4. Haines JL, Hauser MA, Schmidt S, et al. Complement factor $\mathrm{H}$ variant increases the risk of age-related macular degeneration. Science. 2005;308(5720):419-421.

5. Klein RJ, Zeiss C, Chew EY, et al. Complement factor $\mathrm{H}$ polymorphism in age-related macular degeneration. Science. 2005;308(5720):385-389.

6. Gold B, Merriam JE, Zernant J, et al. Variation in factor B (BF) and complement component 2 (C2) genes is associated with age-related macular degeneration. Nat Genet. 2006;38(4):458462.

7. Yates JR, Sepp T, Matharu BK, et al. Complement C3 variant and the risk of age-related macular degeneration. N Engl J Med. 2007;357(6):553-561.

8. Hogg RE, Woodside JV, Gilchrist SE, et al. Cardiovascular disease and hypertension are strong risk factors for choroidal neovascularization. Ophthalmology. 2008;115(6):1046-1052 e1042.

9. Seddon JM, George S, Rosner B, Rifai N. Progression of age-related macular degeneration: prospective assessment of C-reactive protein, interleukin 6 , and other cardiovascular biomarkers. Arch Ophthalmol. 2005;123(6):774-782.

10. Wang JJ, Rochtchina E, Smith W, et al. Combined effects of complement factor $\mathrm{H}$ genotypes, fish consumption, and inflammatory markers on long-term risk for age-related macular degeneration in a cohort. Am J Epidemiol. 2009;169(5):633-641.

11. Wu KH, Tan AG, Rochtchina $E$, et al. Circulating inflammatory markers and hemostatic factors in age-related maculopathy: a population-based case-control study. Invest Ophthalmol Vis Sci. 2007;48(5):1983-1988.

12. Reynolds R, Hartnett ME, Atkinson JP, Giclas PC, Rosner B, Seddon JM. Plasma complement components and activation fragments: associations with age-related macular degeneration genotypes and phenotypes. Invest Ophthalmol Vis Sci. 2009;50(12):5818-5827.

13. Salazar J, Martinez MS, Chavez M, et al. C-reactive protein: clinical and epidemiological perspectives. Cardiol Res Pract. 2014:605810.

14. Hong T, Tan AG, Mitchell P, Wang JJ. A review and meta-analysis of the association between C-reactive protein and age-related macular degeneration. Surv Ophthalmol. 2011;56(3):184194.

15. Mitta VP, Christen WG, Glynn RJ, et al. C-reactive protein and the incidence of macular degeneration: pooled analysis of 5 cohorts. JAMA Ophthalmol. 2013;131(4):507-513.

16. Emerging Risk Factors Collaboration, Kaptoge S, Di Angelantonio E, et al. C-reactive protein concentration and risk of coronary heart disease, stroke, and mortality: an individual participant meta-analysis. Lancet. 2010;375(9709):132-140.

17. Wensley F, Gao P, Burgess $\mathrm{S}$, et al. Association between $\mathrm{C}$ reactive protein and coronary heart disease: mendelian randomisation analysis based on individual participant data. $\mathrm{Br}$ Med J. 2011;342.

18. Elliott P, Chambers JC, Zhang W, et al. Genetic Loci associated with C-reactive protein levels and risk of coronary heart disease. JAMA. 2009;302(1):37-48.

19. Smith GD, Ebrahim S. What can mendelian randomisation tell us about modifiable behavioural and environmental exposures? Br Med J. 2005;330(7499):1076-1079. 
20. Carlson CS, Aldred SF, Lee PK, et al. Polymorphisms within the C-reactive protein (CRP) promoter region are associated with plasma CRP levels. Am J Hum Genet. 2005;77(1):64-77.

21. CRP CHD Genetics Collaboration. Collaborative pooled analysis of data on C-reactive protein gene variants and coronary disease: judging causality by Mendelian randomisation. Eur J Epidemiol. 2008;23(8):531-540.

22. Kim IK, Ji F, Morrison MA, et al. Comprehensive analysis of CRP, CFH Y402H and environmental risk factors on risk of neovascular age-related macular degeneration. Mol Vis. 2008;14:1487-1495.

23. Despriet DDG, Klaver CCW, Witteman JCM, et al. Complement factor H polymorphism, complement activators, and risk of age-related macular degeneration. JAMA. 2006;296(3):301-309.

24. Schaumberg DA, Christen WG, Kozlowski P, Miller DT, Ridker PM, Zee RYL. A prospective assessment of the $\mathrm{Y} 402 \mathrm{H}$ variant in complement factor $\mathrm{H}$, genetic variants in C-reactive protein, and risk of age-related macular degeneration. Invest Ophthalmol Vis Sci. 2006;47(6):2336-2340.

25. Augood C, Fletcher A, Bentham G, et al. Methods for a population-based study of the prevalence of and risk factors for age-related maculopathy and macular degeneration in elderly European populations: the EUREYE study. Ophthalmic Epidemiol. 2004;11(2):117129.

26. Sofat R, Casas JP, Webster AR, et al. Complement factor $\mathrm{H}$ genetic variant and age-related macular degeneration: effect size, modifiers and relationship to disease subtype. Int J Epidemiol. 2012;41(1):250-262.

27. Bird AC, Bressler NM, Bressler SB, et al. An international classification and grading system for age-related maculopathy and age-related macular degeneration. The International ARM Epidemiological Study Group. Surv Ophthalmol. 1995;39(5):367-374.

28. Pearson TA, Mensah GA, Alexander RW, et al. Markers of inflammation and cardiovascular disease: application to clinical and public health practice: A statement for healthcare professionals from the Centers for Disease Control and Prevention and the American Heart Association. Circulation. 2003;107(3):499-511.

29. Verzilli C, Shah T, Casas JP, et al. Bayesian meta-analysis of genetic association studies with different sets of markers. Am J Hum Genet. 2008;82(4):859-872.

30. Nimptsch $\mathrm{K}$, Aleksandrova $\mathrm{K}$, Boeing $\mathrm{H}$, et al. Association of CRP genetic variants with blood concentrations of C-reactive protein and colorectal cancer risk. Int J Cancer. 2014.

31. Fritsche LG, Chen W, Schu M, et al. Seven new loci associated with age-related macular degeneration. Nat Genet. 2013;45(4):433-439, 439e431-432.

32. Fritsche LG, IgI W, Bailey JN, et al. A large genome-wide association study of age-related macular degeneration highlights contributions of rare and common variants. Nat Genet. 2016;48(2):134-143.

33. Ulas F, Balbaba M, Ozmen S, Celebi S, Dogan U. Association of dehydroepiandrosterone sulfate, serum lipids, C-reactive protein and body mass index with age-related macular degeneration. Int Ophthalmol. 2013;33(5):485-491.

34. Klein $\mathrm{R}$, Knudtson $\mathrm{MD}$, Klein $\mathrm{BE}$, et al. Inflammation, complement factor $\mathrm{h}$, and age-related macular degeneration: the Multi-ethnic Study of Atherosclerosis. Ophthalmology. 2008;115(10):1742-1749.

35. McGwin G, Hall TA, Xie A, Owsley C. The relation between $C$ reactive protein and age related macular degeneration in the Cardiovascular Health Study. Br J Ophthalmol. 2005;89(9):11661170.

36. Klein R, Klein BE, Marino EK, et al. Early age-related maculopathy in the cardiovascular health study. Ophthalmology. 2003;110(1):25-33. 
37. Yip JL, Khawaja AP, Chan MP, et al. Cross Sectional and Longitudinal Associations between Cardiovascular Risk Factors and Age Related Macular Degeneration in the EPIC-Norfolk Eye Study. PLoS One. 2015;10(7):e0132565.

38. Molins B, Fuentes-Prior P, Adan A, et al. Complement factor $\mathrm{H}$ binding of monomeric $\mathrm{C}$ reactive protein downregulates proinflammatory activity and is impaired with at risk polymorphic CFH variants. Sci Rep. 2016;6.

39. Dehghan A, Dupuis J, Barbalic M, et al. Meta-analysis of genome-wide association studies in $>80000$ subjects identifies multiple loci for C-reactive protein levels. Circulation. 2011;123(7):731-738.

40. McKay GJ, Patterson CC, Chakravarthy U, et al. Evidence of association of APOE with agerelated macular degeneration: a pooled analysis of 15 studies. Hum Mutat. 2011;32(12):1407-1416. 